\title{
Population structure and spatio-temporal transmission dynamics of Plasmodium vivax after radical cure treatment in a rural village of the Peruvian Amazon
}

Christopher Delgado-Ratto ${ }^{1,2^{*}}$, Veronica E Soto-Calle ${ }^{3}$, Peter Van den Eede ${ }^{4}$, Dionicia Gamboa ${ }^{2,5}$, Angel Rosas ${ }^{3,4}$, Emmanuel N Abatih ${ }^{6}$, Hugo Rodriguez Ferrucci ${ }^{7}$, Alejandro Llanos-Cuentas ${ }^{3}$, Jean-Pierre Van Geertruyden ${ }^{1}$, Annette Erhart ${ }^{4}$ and Umberto D'Alessandro ${ }^{8,9}$

\begin{abstract}
Background: Despite the large burden of Plasmodium vivax, little is known about its transmission dynamics. This study explored the population structure and spatio-temporal dynamics of $P$. vivax recurrent infections after radical cure in a two-year cohort study carried out in a rural community of the Peruvian Amazon.

Methods: A total of 37 P. vivax participants recruited in San Carlos community (Peru) between April and December 2008 were treated radically with chloroquine and primaquine and followed up monthly for two years with systematic blood sampling. All samples were screened for malaria parasites and subsequently all $P$. vivax infections genotyped using 15 microsatellites. Parasite population structure and dynamics were determined by computing different genetic indices and using spatio-temporal statistics.
\end{abstract}

Results: After radical cure, $76 \%$ of the study participants experienced one or more recurrent $P$. vivax infections, most of them sub-patent and asymptomatic. The parasite population displayed limited genetic diversity $(\mathrm{He}=0.49)$ and clonal structure, with most infections (84\%) being monoclonal. Spatio-temporal clusters of specific haplotypes were found throughout the study and persistence of highly frequent haplotypes were observed over several months within the same participants/households.

Conclusions: In San Carlos community, P. vivax recurrences were commonly observed after radical treatment, and characterized by asymptomatic, sub-patent and clustered infections (within and between individuals from a few neighbouring households). Moreover low genetic diversity as well as parasite inbreeding are likely to define a clonal parasite population which has important implications on the malaria epidemiology of the study area.

Keywords: Plasmodium vivax, Malaria, Genotyping, Microsatellites, Population genetics, Spatio-temporal analysis, Peruvian amazon

\footnotetext{
* Correspondence: richardchristopher.delgadoratto@uantwerpen.be

'Unit of International Health, ESOC Department, Faculty of Medicine, University of Antwerp, Universiteitsplein 1, B-2610 Antwerp, Belgium

${ }^{2}$ Laboratory of Malaria, Unit of Molecular Epidemiology, Institute of Tropical Medicine Alexander von Humboldt (IMTAvH)-Universidad Peruana Cayetano Heredia (UPCH), Av Honorio Delgado 430, Lima, Peru

Full list of author information is available at the end of the article
} 


\section{Background}

Plasmodium vivax, the most widely distributed malaria species, threatens almost $40 \%$ of the worldwide population [1]. Vivax malaria is less benign than commonly thought [1] and, due to its complex life cycle, which includes liver hypnozoites that can be re-activated well after the primary infection, it is difficult to have a clear picture of its burden and epidemiology. Limited knowledge of the underlying $P$. vivax population structure and dynamics are major obstacles to achieve effective malaria control, particularly in low endemic areas [2]. In the Peruvian Amazon, vivax malaria is still a major public health problem [3]. Both chloroquine-resistant strains [4] and the frequent occurrence of recurrent infections after 'radical' treatment [chloroquine (CQ) and primaquine (PQ)] have been reported $[4,5]$. Moreover, the frequent occurrence of clustered asymptomatic infections makes malaria control in this low endemic setting extremely difficult [6-8]. Therefore, there is the need to carry out $P$. vivax population genetic studies to understand how parasites are circulating in the human population, as well as related topics such as the spread of drug resistance and the development of clinical immunity $[9,10]$. Similar to other endemic Amazonian countries [11-13], P. vivax populations in the Peruvian Amazon are extremely heterogeneous, with different degrees of diversity and polyclonality $[14,15]$. This study was part of a larger ongoing $P$. vivax cohort conducted within 21 rural communities, and which results will be published later. In this paper, one of these study villages has been selected in order to analyse, at community and household level, the population structure and the spatialtemporal dynamics of $P$. vivax infections after radical treatment of the primary infection.

\section{Methods}

Study site

The study was conducted in San Carlos (latitude $3^{\circ} 57^{\prime}$ $30.99^{\prime \prime} \mathrm{S}$ longitude $\left.73^{\circ} 20^{\prime} 45.76^{\prime \prime} \mathrm{W}\right)$, a relatively small and isolated village in the Peruvian Amazon, situated at $10 \mathrm{~km}$ from the main road (Iquitos-Nauta road) and $21 \mathrm{~km}$ southeast of Iquitos city (six hours by boat via the Itaya river) [14] (Figure 1). Malaria transmission is perennial with a peak during the rainy season (NovemberMay). About $80 \%$ of malaria cases are caused by $P$. vivax, and Anopheles darlingi is the main vector $[7,16]$. The 127 habitants of San Carlos (2008 census) were primarily 'mestizos' (individuals not belonging to a specific ethnic minority) living in open wooden houses (average of 4.7 persons/house), mainly occupied in slash and burn agriculture and small scale fishery [17]. Households are situated close (13-45 m) to each other with the maximal distance between any two households being of less than $1 \mathrm{~km}$ (V Soto-Calle, pers comm). Neither electricity nor

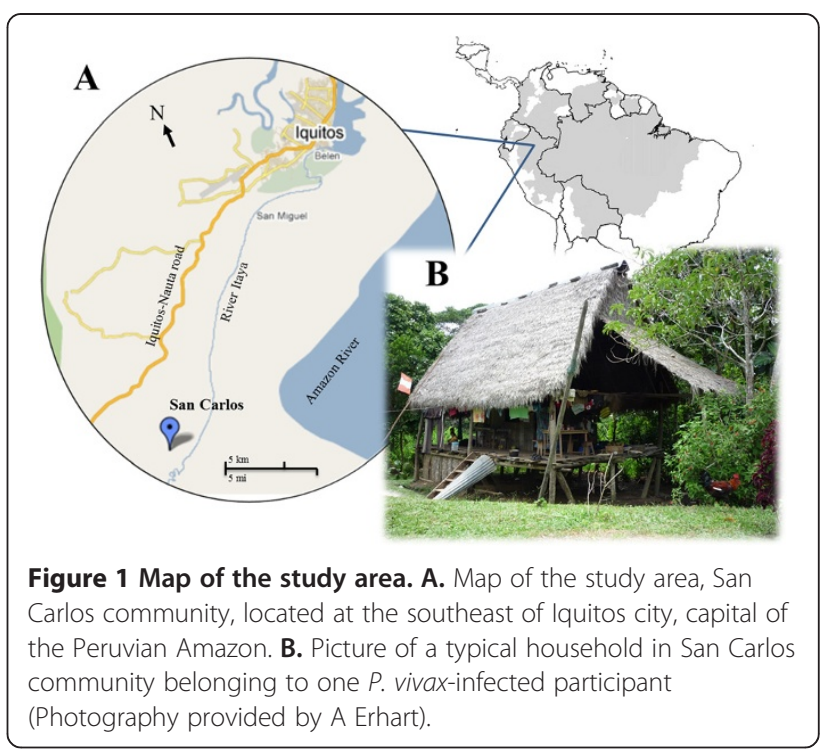

basic sanitation services are available, and the closest health post was approximately $3 \mathrm{~km}$ away from San Carlos community.

\section{Sample collection}

Plasmodium vivax-infected participants were identified by microscopy examination of blood smears collected during an initial screening of the village population (April 2008) followed by active case detection of all fever cases (April to December 2008), following the study protocol of the larger cohort study. All microscopically confirmed $P$. vivax-infected participants were treated (directly observed treatment) with CQ $25 \mathrm{mg} / \mathrm{kg} /$ day (three days) and PQ $0.50 \mathrm{mg} / \mathrm{kg} /$ day (seven days), following the national guidelines for radical cure [5]. The most important limitation for the seven-day regimen in the Peruvian Amazon is the adherence to the treatment [18]. For this reason, the treatment was administered in a supervised way by a health care worker (hired exclusively for this study) and monitored every day during the seven-day treatment. Participants were asked their written informed consent (parents/guardians for minors) to be monthly follow-up for two years in order to detect $P$. vivax recurrent infections. Study subjects were visited at home weekly for the first month after treatment, and then monthly for the next 23 months. If a participant missed one scheduled sampling, the health care worker came back to the community two more times to look for the participant and/or scheduled the most feasible time to visit. At each visit, the medical staff asked study participants about any symptoms they may have had since the previous visit. A blood sample for microscopy (thick and thin film) and a blood spot on filter paper (BSFP) (Whatman grade 3, Whatman, Springfield Mill, USA) for later molecular analysis were systematically collected. 
Between scheduled visits, participants were encouraged to consult the study team whenever they felt ill, to check for fever and malaria infection (blood smear and BSFP). If malaria parasites were identified by microscopy in the field after recruitment, participants were treated with CQ for three days under direct supervision, and followed up until parasite clearance (confirmed by microscopy). Those participants with 2 or more episodes of malaria after the recruitment were given a complete 7-day PQ treatment before the 2 year follow up. This study was approved by the Ethical Boards of Universidad Peruana Cayetano Heredia, Peru (Project PVIVAX-UPCH, SIDISI code: 053256), the Institute of Tropical Medicine Antwerp and the University Hospital of Antwerp (Belgium).

\section{Laboratory methods}

All day 0 (D0) blood samples were analysed by microscopy (thin and thick film) and Plasmodium species-specific PCR (ssPCR) to confirm the presence and density of $P$. vivax parasites, and this was repeated for all blood samples taken during participants' monthly follow-up (scheduled and unscheduled visits). Parasite density was computed after counting the number of asexual parasites for 200 white blood cells (WBC) in the thick smear and assuming a concentration of $8,000 \mathrm{WBCs} / \mu \mathrm{l}$. A slide was declared negative if no malaria parasite was found after examining 100 fields. Quality control was done by a senior technician on all positive and $10 \%$ of negative slides [19].

Parasite DNA was extracted from the BSFP using the saponin lysis Chelex 100 method [20], and either stored at $4^{\circ} \mathrm{C}$ for immediate use in PCR reactions or at $-20^{\circ} \mathrm{C}$ for later use. All samples were first analysed by ssPCR following a protocol published elsewhere [21]. Briefly, this ssPCR consisted of a primary PCR with primers directed to specific Plasmodium and mammal sequences, and obtained amplicons were used for a second PCR using Plasmodium-species specific primers.

Positive vivax malaria samples, either by microscopy and/or species specific PCR, were selected for genotyping using 15 neutral, or nearly neutral, microsatellite markers (MS) as described elsewhere [22,23]. This technique consists of PCR amplification of 15 microsatellite sequences using for each reaction two specific pairs of primers (flurophore-labelled forward primer and no labelled reverse primer). The PCR fragments were analysed by capillary electrophoresis in a $3730 \mathrm{XL}$ ABI sequencer (Applied Biosystems, Foster City, CA, USA). The size of the PCR fragments was determined using Genemapper (Applied Biosystems, Foster City, CA, USA); bands smaller than 100 relative fluorescence units (RFU) were defined as background. Samples presenting no microsatellite PCR (MS PCR) amplification in some loci were re-analysed [23].

\section{Data analysis}

Data were double entered and cleaned in Excel (Microsoft, USA) and then analysed with SPSS v.16 (SPSS Inc, Chicago, IL, USA). A $P$. vivax recurrence was defined as any symptomatic (i e, fever: axillary temperature $>37.5^{\circ} \mathrm{C}$ ) or asymptomatic $P$. vivax infection identified by microscopy and/or PCR occurring between seven days and 24 months after the start of radical cure treatment. Recurrences were defined as "patent" if identified by microscopy, and "sub-patent" if detected only by ssPCR. All sub-patent infections were assumed to have an average parasite density of $10 / \mu \mathrm{l}$ [24]. The overall mean parasite density (geometric means) at day zero (D0) and overall density at monthly follow-up visits were compared by Student's $t$-test.

\section{Genetic structure and population dynamics}

An infection was defined as polyclonal (presence of two or more genetically distinct clones) if at least one locus presented more than one allele [25]. The percentage of polyclonal samples detected by each locus was described. The locus with the highest number of alleles was considered as a proxy for the 'multiplicity of infection' (MOI), representing the minimal number of parasite haplotypes in the sample [14]. Haplotypes, described as unique allelic combinations of the 15 loci analysed, were determined in monoclonal samples using Genclone v2.0 [26]. The haplotypes within polyclonal samples were analysed using both the predominant and minor alleles in the sample (peak size in the electropherogram) and defined respectively as the most probable dominant and minor clones. The proportions of monoclonal/polyclonal infections in children $(\leq 14$ years old) and adults $(>14$ years old) at D0 and among recurrent infections were compared using Pearson chi-square test.

The total number of alleles per each locus, the allelic richness (number of alleles per locus independently of the sample size), the number of alleles detected in a sample by any locus, and the genetic diversity of each locus (expected heterozygosity $(\mathrm{He})$ ) were computed for each population using FSTAT v.2.9.3 [27]. The He represents the probability of finding a different allele for a given locus in any pair of haploids randomly drawn from the same population. Genepop v4.0 software was used to assess the population differentiation through the analysis of the allele distribution $[28,29]$.

The standardized index of association $\left(I_{A}^{s}\right)$ was computed with LIAN 3.5 software [30] and used to test whether multilocus linkage disequilibrium (multilocus LD) occurred in local parasite population $[11,12,14]$. The $I_{A}^{s}$ was assessed among monoclonal infections, respectively for D0, monthly recurrences and all samples together. In addition, pair-wise linkage disequilibrium (pair-wise LD) was assessed between every pair of 
markers using G-statistics with FSTAT software [27] to avoid any bias due to physical linkage between particular loci located within the same contigs (relatively long segments of contiguous DNA sequence assembled during genome analysis) [11]. The probability of finding identical haplotypes derived from distinct sexual reproductive events $\left(p_{\text {sex }}\right)$ in two or more samples was computed using GenClone ver. 2.0 [26].

Three different approaches (eBURST, STRUCTURE and NETWORK) were used to identify clonal parasite groups. eBURST software v.3 [31] was used to identify haplogroups, defined as clusters of closely related haplotypes that are identical to each other on at least 11 loci [13]. The haplotypes that were not related to any haplogroup were classified as singletons. The haplogroups and singletons were used to analyse the transmission dynamics of the parasites at the genetic level over time [11,13]. In addition, STRUCTURE v.2.3.3 was used to identify distinct sub-populations and determine fractions of the haplotype for each strain that belong to each subpopulation. The most probable number of clusters was defined by calculating the $K$ value as described elsewhere [32]. The relationship between $P$. vivax haplotypes was further analysed by a simple phylogenetic approach using software Network v.4.6.1.0 [33].

Using the geo-coding of the participants at household level and the SaTScan software v9.1.1 [34], space-time scan statistics were employed to identify clusters of space-time malaria cases with elevated proportions of specific haplotypes. This was done by gradually scanning a window across time and space, noting the number of observed and expected infections inside the window at each location. The space-time clusters were assessed with up to a maximum of $50 \%$ of the data included in the scanning window and a maximum temporal window size of up to $50 \%$ of the study period. The relative risk (RR) was computed by dividing the estimated risk within the cluster by the estimated risk outside the cluster. The window with the maximum likelihood is defined as the most likely cluster. The significance of the identified space-time clusters was tested using the likelihood ratio test statistic and $p$-values were obtained through Monte Carlo simulations [35].

\section{Results}

\section{Baseline characteristics}

In April 2008 (screening), the prevalence of vivax malaria in San Carlos was estimated at $10.2 \%$ by standard microscopy. Between April and December 2008, a total of 37 individuals (in 20 households) were identified with a $P$. vivax mono-infection and recruited into the study. Males $(n=19)$ and females $(n=18)$ were equally represented, and the median age was 15 years (IQR 3-48) with $40 \%$ of study participants aged $<$ nine years. At D0 the mean parasite density was $2,031 / \mu \mathrm{l}(95 \% \mathrm{CI}: 1,332$ 3,098). Though all D0 slides were double-read by a senior technician, the ssPCR remained negative (ssPCR repeated once after negative amplification) for two participants whose parasite density by microscopy was $48 / \mu \mathrm{l}$ and $823 / \mu \mathrm{l}$, respectively. During the first 28 days of follow-up, there was no $P$. vivax recurrence observed by microscopy or by ssPCR (Figure 2).

\section{Plasmodium vivax recurrences during the monthly follow-up}

At day 28, all 37 participants were found negative both by microscopy and ssPCR. During the following 23month period, a total of $142 P$. vivax recurrent infections were detected by microscopy with a mean parasite density of $88 / \mu \mathrm{l}$ (95\% CI: $56-139)$ which was significantly lower than that at D0 $(\mathrm{p}<0.001)$. The ssPCR missed two recurrent infections despite a parasite density by microscopy of 36 and $4,352 / \mu \mathrm{l}$, respectively. By the end of the two-year follow-up, 76\% (28/37) of the study subjects had experienced at least one recurrent $P$. vivax infection, while only two Plasmodium falciparum infections were identified. More than half of the recurrent infections $(54.9 \%, 78 / 142)$ were sub-patent and asymptomatic.

\section{Genetic diversity of the Plasmodium vivax population}

A total of 174 P. vivax infections (35 D0 and 139 recurrences) were genotyped by MS PCR (Figure 2), among which 164 had a MS PCR amplification. Most of them $(82.9 \%, 136 / 164)$ were monoclonal and had complete allele data (for all loci) $(91.9 \%, 125 / 136)$. The proportions of monoclonal and polyclonal infections did not differ between baseline and recurrent infections, and neither did the average multiplicity of infection (MOI) (Table 1). The average expected heterozygosity $(\mathrm{He})$ was 0.49 (range $0.10-0.67$ ) (Table 1), and MS4 was the most informative marker $(\mathrm{He}=0.65)$ discriminating $58.6 \%$ of all polyclonal infections. The average total number of alleles per locus was 4.6 (range 2-7), the average allelic richness was 4.17 (range 1.99-6.28) and the average number of alleles detected in a sample by any locus remained constant between all loci (1.1-1.2). Average genetic indexes did not vary between D0 and recurrences, however looking at specific periods of the study, differences were found, as shown later.

\section{Parasite inbreeding}

The analysis of the monoclonal samples $(\mathrm{n}=136)$ showed a highly significant overall LD $\left(I_{A}^{s}=0.51\right.$, $\mathrm{p}<0.0001)$ indicating a clonal population. To check for bias due to unequal distribution of the haplotypes or due to sampling, the LD was also assessed for unique haplotypes only, and it remained significant $\left(I_{A}^{s}=0.15\right.$, 


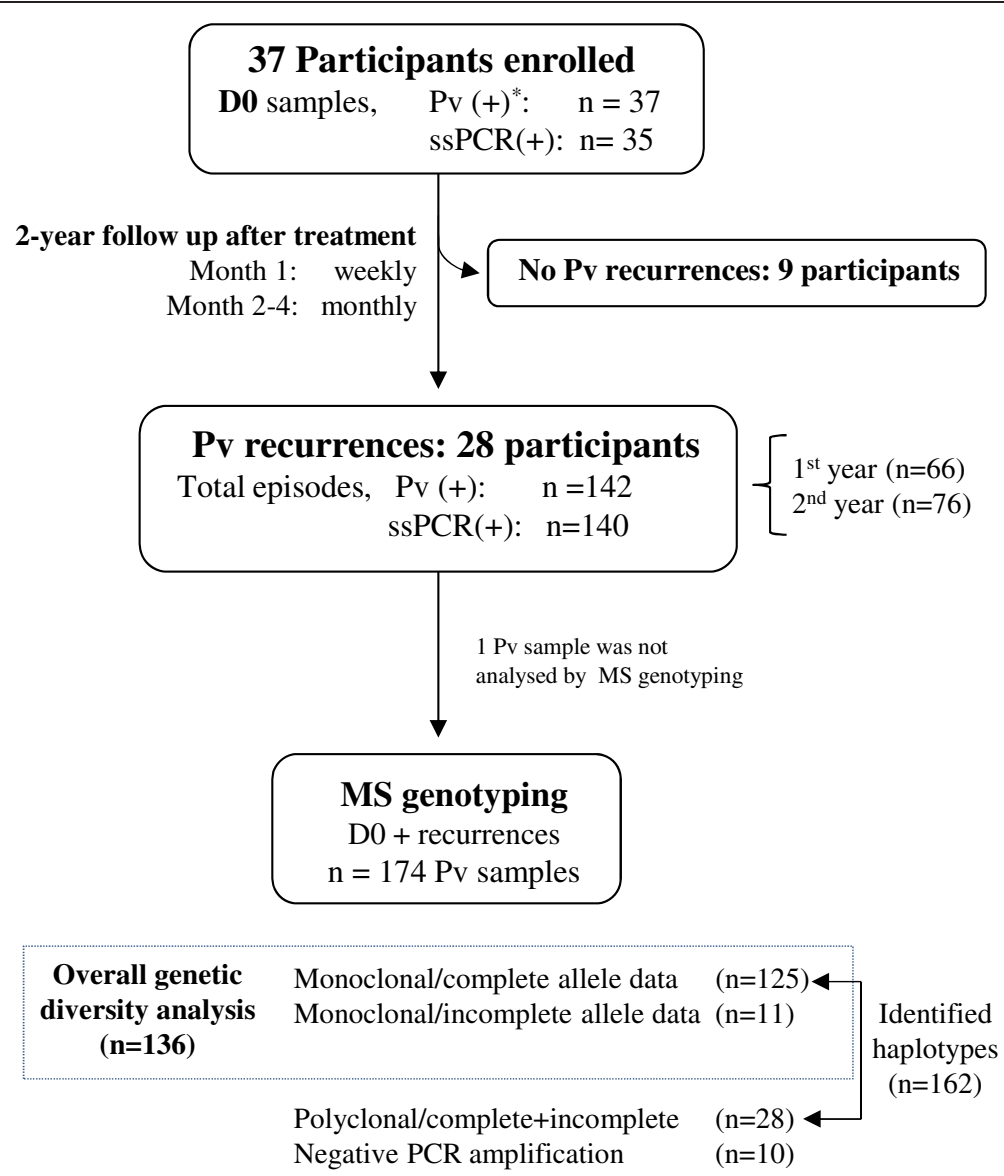

Figure 2 Study flowchart. Thirty-seven participants were enrolled and treated $(\mathrm{CQ}+\mathrm{PQ})$ on $\mathrm{D} 0$. Twenty-eight participants had at least one recurrence and nine participants had no recurrences after treatment. Only samples positive for $P$. vivax by ssPCR were genotyped with 15 microsatellites (MS). In total, 162 haplotypes were detected in monoclonal and polyclonal $P$. vivax samples. From 28 polyclonal infections only 37 haplotypes were identified as described in the Methods section. ${ }^{*}=$ microscopically positive. Pv $=P$. vivax.

$\mathrm{p}<0.0001$ ). Inbreeding, rather than physical linkage between markers established in the same contig (MS4-MS5, MS7-MS8 and MS12-MS15), was identified by the linkage between multiple loci obtained by pair-wise LD analysis (Figure 3). Indeed, the probability that the clonal population was due to reproductive events between genetically unrelated parents was very low $\left(p_{s e x}=0.0006\right)$.

\section{Plasmodium vivax population structure}

A total of 162 haplotypes were identified, 125 from monoclonal and 37 from polyclonal infections, of which 20 were classified as unique haplotypes (Figures 2 and 4). $\mathrm{H} 11, \mathrm{H} 4$ and $\mathrm{H} 5$ were the most frequent haplotypes detected in 45,15 and $16 \%$ of the infections, respectively. Moreover, eight haplotypes were detected two to five times $(<3 \%$ each) and nine haplotypes were detected only once. Using eBURST (criteria $=11$ common loci), four haplogroups (A, B, C, and D) containing two to ten unique haplotypes and three singletons were identified. Similarly to eBURST, four populations $(k=4)$ were inferred using STRUCTURE and were very similar to the haplogroups previously defined by eBURST (Figure 4 and Additional file 1). The phylogenetic tree obtained using NETWORK supported the classification results obtained by eBURST: i) haplogroup A contained ten low frequency, slightly different haplotypes; ii) haplogroup B, derived from haplogroup A, contained only two haplotypes (two extra haplotypes, $\mathrm{H} 8$ and $\mathrm{H} 18$, considered as singletons by eBURST belonged to this group following the classification by STRUCTURE (Pop. B); and, iii) and iv) haplogroup $\mathrm{C}$ and $\mathrm{D}$ contained two to three haplotypes but were dominant throughout the study (Figure 4).

\section{Parasite dynamics and space-time clustering}

The genetic diversity $\mathrm{He}$ ) did not vary substantially between baseline (D0) and first year monthly follow-up samples, 0.44 and $0.40(\mathrm{p}=0.26)$, respectively. Nevertheless, $\mathrm{He}$ was significantly higher during the second year of follow-up $(\mathrm{He}=0.51)$ compared to both baseline and first year $(\mathrm{p}<0.0001)$. In addition, compared to the first 
Table 1 Overall polymorphism of the 15 Plasmodium vivax microsatellite loci assessed in San Carlos community

\begin{tabular}{lllllll}
\hline Locus & Total number of alleles & Allele size (range in bp) & He & Allelic richness & Polyclonal samples/locus (\%) & Average alleles/locus \\
\hline MS1 & 4 & $234-242$ & 0.1 & 2.94 & $0 \%$ & 1.1 \\
MS2 & 5 & $187-207$ & 0.6 & 4.89 & $13.8 \%$ & 1.1 \\
MS3 & 2 & $181-184$ & 0.13 & 1.99 & $6.9 \%$ & 1.1 \\
MS4 & 5 & $165-198$ & 0.65 & 4.62 & $58.6 \%$ & 1.2 \\
MS5 & 2 & $166-172$ & 0.29 & 2 & 1.1 \\
MS6 & 5 & $208-244$ & 0.61 & 4.38 & $17.2 \%$ & 1.1 \\
MS7 & 3 & $142-148$ & 0.47 & 2.98 & $17.2 \%$ & 1.1 \\
MS8 & 4 & $252-271$ & 0.6 & 4 & $24.1 \%$ & 1.1 \\
MS9 & 5 & $155-172$ & 0.66 & 4.94 & $20.7 \%$ & 1.1 \\
MS10 & 4 & $210-266$ & 0.13 & 3.19 & $0 \%$ & 1.1 \\
MS12 & 6 & $181-332$ & 0.55 & 5.14 & $27.6 \%$ & 1.1 \\
MS15 & 4 & $210-256$ & 0.59 & 3.98 & $31 \%$ & 1.1 \\
MS16 & 7 & $193-350$ & 0.67 & 5.92 & $13.8 \%$ & 1.1 \\
MS20 & 7 & $194-241$ & 0.65 & 6.28 & $24.1 \%$ & 1.1 \\
Pv6635 & 6 & $184-202$ & 0.67 & 5.34 & $27.6 \%$ & 1.1
\end{tabular}

A total of 174 P. vivax infections were analysed, including 35 D0 and 139 recurrent samples. The expected heterozygosity (He) and the allelic richness were calculated only for monoclonal samples $(n=136)$.

year, multilocus LD $\left(I_{A}^{s}\right)$ during the second year decreased from 0.6 to 0.4 . The frequency of the most common haplotype at D0 and during the first year, i e, H11 (=haplogroup $\mathrm{D}$ ), decreased substantially during the second year (from 60 to $30 \%, \mathrm{p}<0.01$ ), while in the same period new infrequent haplotypes (i e, H1, H3) and other haplotypes belonging to the haplogroup $\mathrm{A}$ were observed (Figure 5, panel A). The frequency of the haplogroup $C$ remained constant throughout the study although its composition varied by year, $\mathrm{H} 4$ gradually replacing H5 during the second year (Figure 5, panel B).
Using SaTScan, a total of six space-time clusters of five different haplotypes (out of 20) were identified during the study period (Figure 6). Clusters of H11 and H5 represented the two main clusters within the first year $(\mathrm{RR}=10.1$ and 13.5 respectively; from May to August 2008), while H4 cluster represented a significant large cluster during the second year $(R R=9.9$; from June 2009 to May 2010). In 2009, H11 was defined as a secondary cluster, and together with two other haplotypes (H7, H8) clustered in single households for several months. Due to sparseness of some haplotypes in multiple households, only

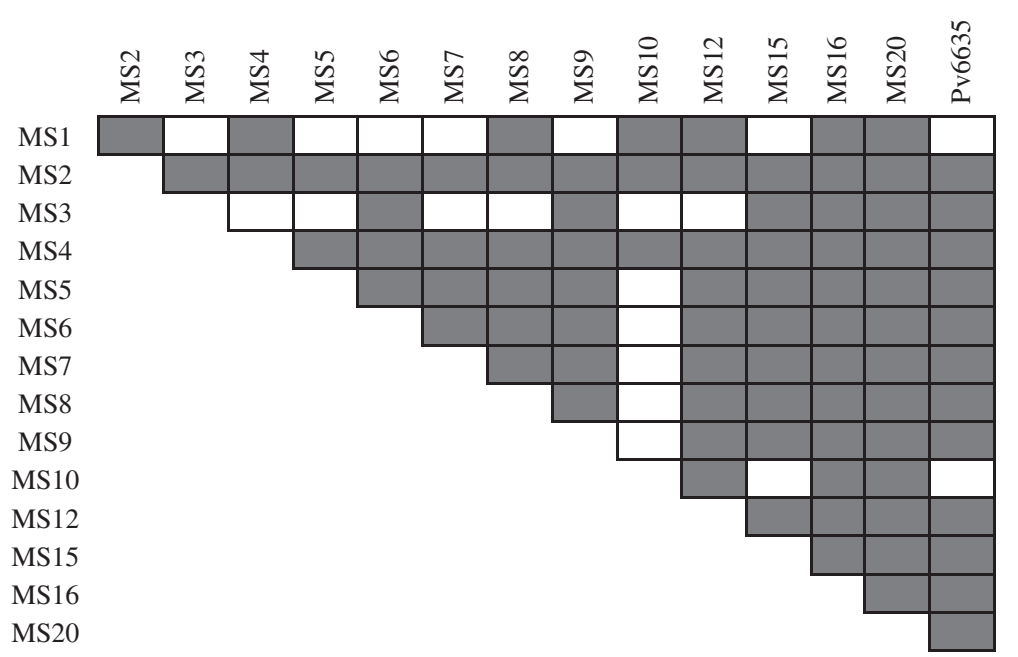

Figure 3 Patterns of linkage disequilibrium between pairs of microsatellite markers in the Plasmodium vivax population from San Carlos community. ( $n=136$ monoclonal samples analysed). Grey shading denotes linkage disequilibrium at the 5\% significance level (adjusted $p=0.00005$ based on $10^{6}$ permutations). 


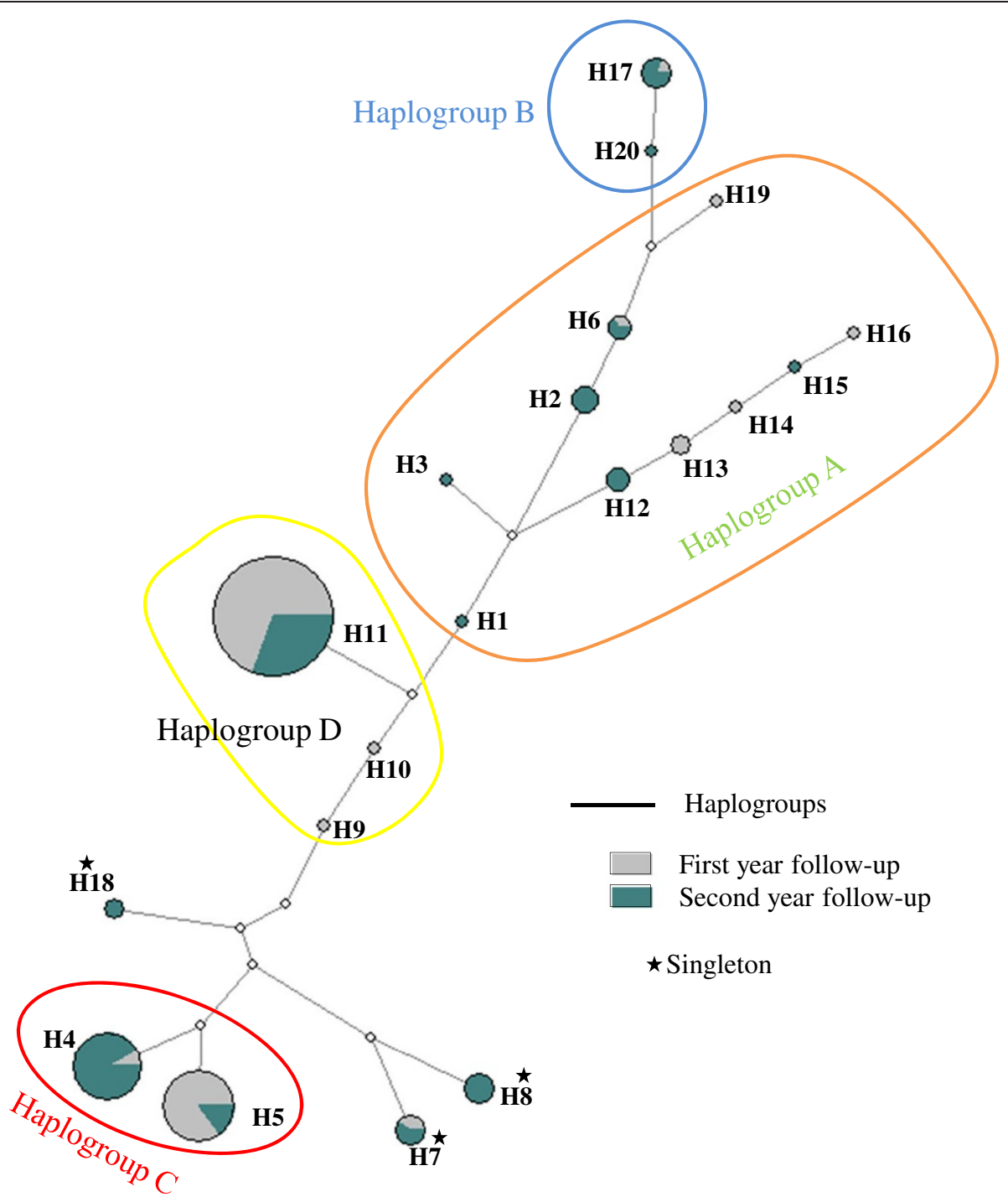

Figure 4 A median joining tree performed using NETWORK representing the relationship between Plasmodium vivax haplotypes and their temporal distribution. Each circle represents a different haplotype and the size reflects the frequency of detection within the two years $(n=162)$. Related haplotypes are linked by a line and mutations (o). Solid lines denote the haplogroups obtained using eBURST v.3 and STRUCTURE v.2.3.3 (Pop A, B, C, D).

clusters with meaningful RR (not zero nor infinity) were reported.

\section{Dynamics at individual level}

More than two thirds of the participants $(68.4 \%, 26 / 37)$ had infections with one to two different haplotypes during their follow-up period, while the remaining had infections with three to five haplotypes (up to three different haplogroups or singletons). The most frequently detected haplotype, H11, dispersed within $80 \%(16 / 20)$ of the participants' households and occurred in $60.7 \%$ (17/28) of the participants in at least one infection (up to eight infections with $\mathrm{H} 11$ in two participants).
One or more sub-patent recurrent infections that cleared without treatment were observed in 50\% (14/28) study subjects (see Additional file 2). Some of these infections had the same haplotype over several consecutive months. During their follow-up, 60\%, (9/15) of study subjects with a H11 haplotype infection at recruitment had several sub-patent recurrent infections with the same haplotype.

\section{Discussion}

In San Carlos, a relatively isolated village in the Peruvian Amazon, the majority (76\%) of the 37 study participants experienced $P$. vivax recurrences after radical cure given according to the national guidelines. Most of the $P$. vivax 

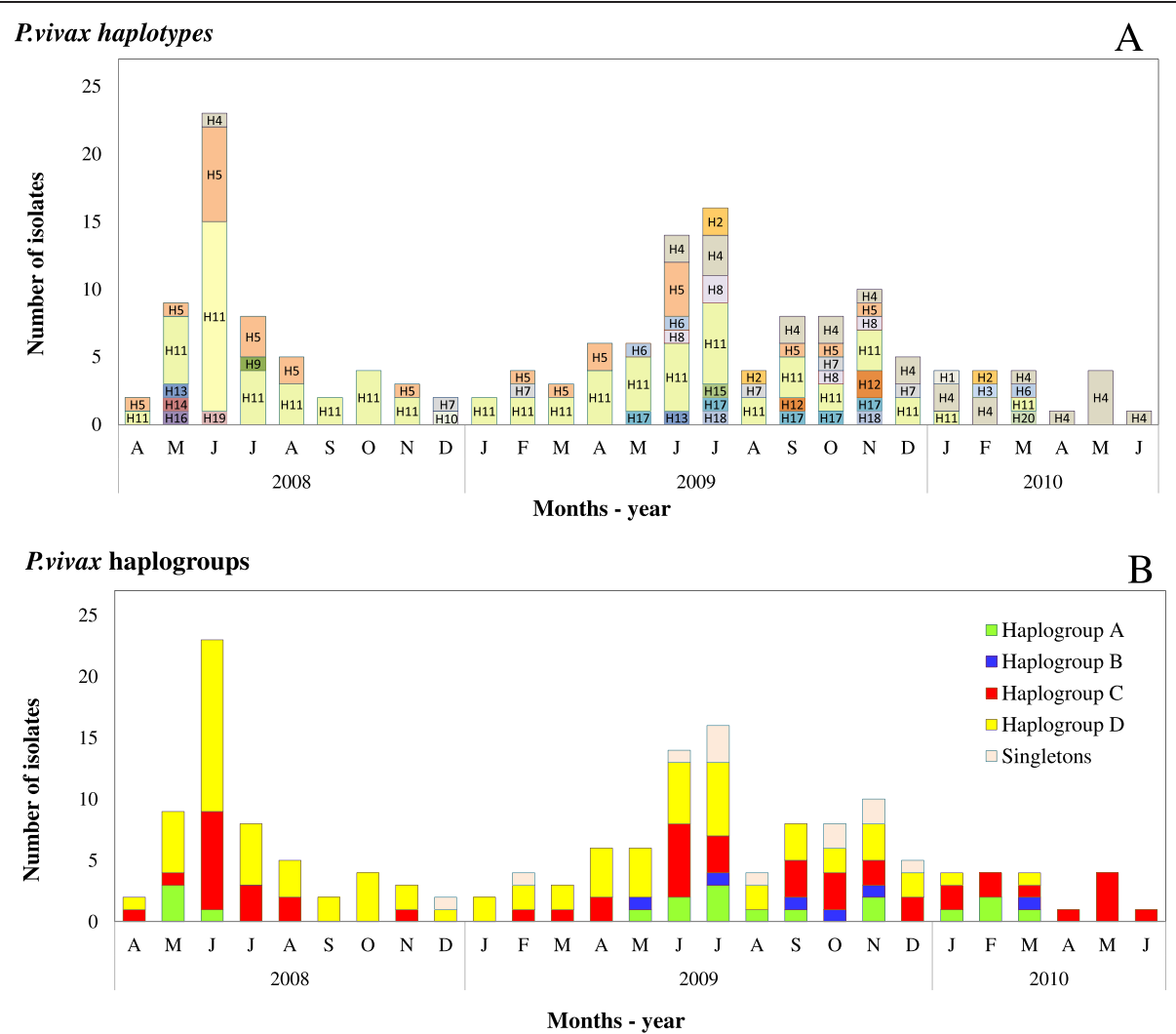

Figure 5 Transmission dynamics of Plasmodium vivax haplotypes and haplogroups in San Carlos community. Panel A: Monthly distribution of the 162 P. vivax haplotypes found among 164 P. vivax infections (patent and sub-patent) between April 2008 and June 2010. Twenty unique haplotypes were detected and are represented by different colours. Panel $\mathbf{B}$ : Monthly distribution of the haplogroups containing closely related haplotypes determined using eBURST. Composition of each haplogroup: A (H1, H2, H3, H6, H12, H13, H14, H15, H16, H19), B (H17, $\mathrm{H} 20), \mathrm{C}(\mathrm{H} 4, \mathrm{H} 5), \mathrm{D}(\mathrm{H} 9, \mathrm{H} 10, \mathrm{H} 11)$, singletons $(\mathrm{H} 7, \mathrm{H} 8, \mathrm{H} 18)$.

recurrences were sub-patent and asymptomatic, some of them persisting for several months. Such a high occurrence of recurrent infections after treatment with a full course of PQ has already been observed in several other South American endemic countries [13,36-39]. The large proportion of monoclonal $P$. vivax infection found in this study was similar to that reported from Colombia [40] but different compared to other communities in the Peruvian Amazon [14] and other areas in South and Central America [11,12]. This is probably due to the differences in effective recombination rates and in human and vector behaviour. The clonality of the parasite population in San Carlos was confirmed by the highly significant LD. The latter can be due to either the low rate of recombination (inbreeding) or the recent occurrence of admixture of two unrelated genotypes that did not have sufficient time to homogenize [41]. Though the latter is theoretically possible, considering the strong differentiation between malaria parasite populations over relatively small geographical distances reported in South America [11,42,43], the low probability of admixture $\left(p_{s e x}\right)$ found in San Carlos indicates that inbreeding was the most likely reproductive event in this area. This was also the case in other reports from endemic areas of the Amazon Basin [11,13,43]. Inbreeding could also explain the replacement of haplotypes without haplogroup replacement as it was observed in this study with the highly prevalent haplogroup C.

Clustering of malaria transmission at household level has been previously reported in the Peruvian Amazon $[6,14]$. In the present study, malaria infections carrying the same haplotype, or haplotypes from the same haplogroup, were identified concurrently or within a period of a few weeks in participants living in the same households. The persistence of infections with the same haplotype for long periods, also due to the relapsing nature of $P$. vivax $[44,45]$, can maintain a certain level of immunity even in low transmission areas like the Amazon Basin [46].

The clustering of haplotypes may also influence the parasite diversity and the interpretation of recurrent parasitaemia. Some participants carried the same haplotype for several consecutive months, and later presented a new but closely related haplotype (Additional file 2). Previous studies have reported also a high rate of 


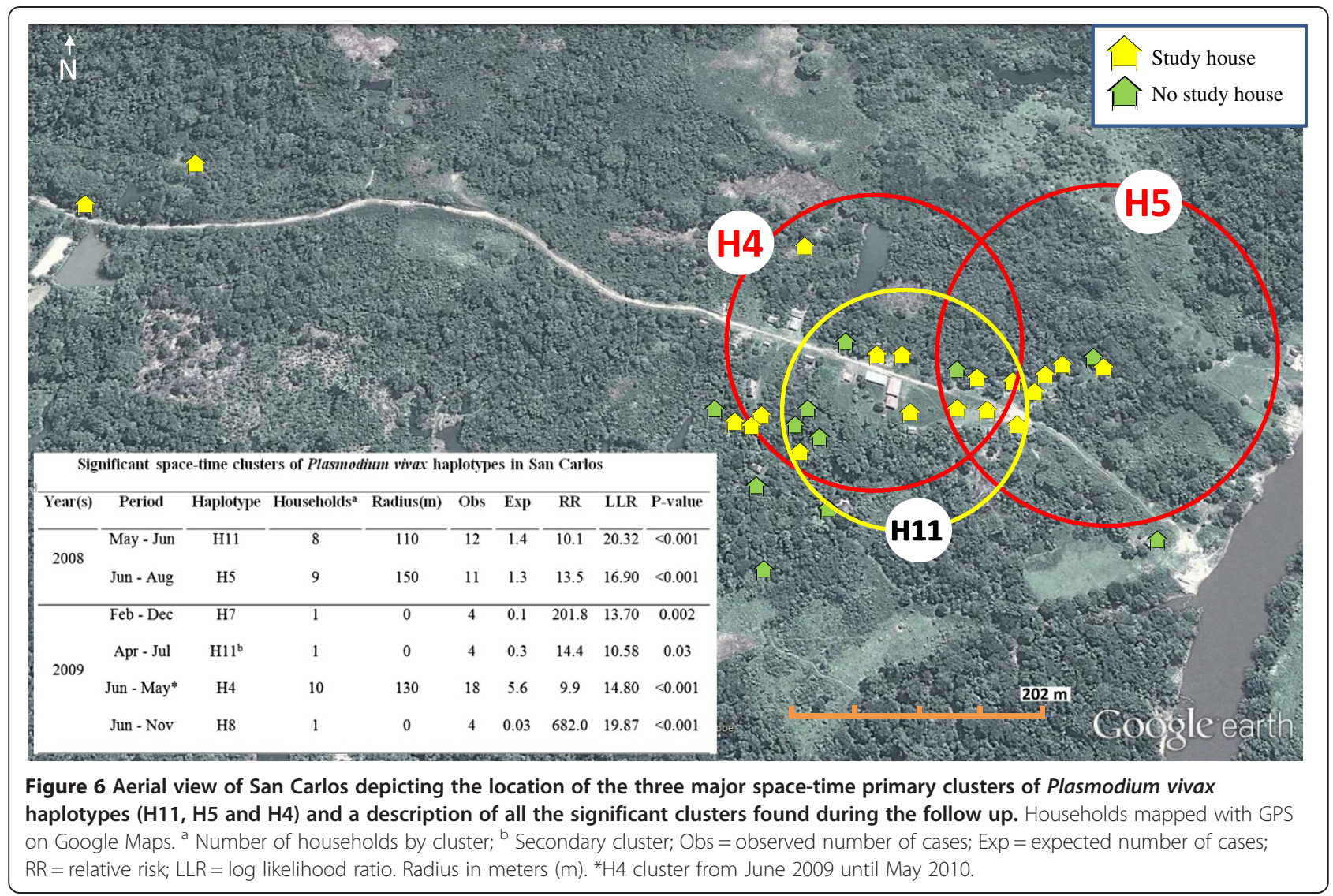

genetically similar recurrent infections in the Peruvian Amazon [44,47]. It was not possible to determine whether this genetic variation originated within the mosquito or the human host. Nevertheless, considering the low transmission in San Carlos, it is likely that most recurrences were not new infections, but rather relapses or recrudescences. For example, haplotypes $\mathrm{H} 4$ and $\mathrm{H} 5$ (distributed in $8 / 20$ and $14 / 20$ households, respectively) infected participants living within the same households (in seven households in total). $\mathrm{H} 4$ and $\mathrm{H} 5$ belong to the same haplogroup and they differ by only one locus (MS8, alleles of 258 and $271 \mathrm{bp}$, respectively). As most of the $P$. vivax microsatellite diversity is produced within the human host during the mitotic replication [48-50] and considering that $\mathrm{H} 4$ displaced $\mathrm{H} 5$ in the second year, deletion of repeat units may have caused the haplotype differentiation in the human host. If this is the case, the recurrence of heterologous parasites may not be necessarily due to previous infection(s) with multiple different clones [51] but to genetic variation of one parasite clone within the human host. In addition, homologous parasites in recurrent infections may represent relapses of a limited diversified parasite population or non-hypnozoite stages (i.e., parasite stages within splenic dendritic cells) that re-activate after extended periods of latency $[5,52,53]$.

\section{Clonal population: possible implications for malaria epidemiology}

Due to the clonality and clustering of the parasite population in San Carlos, people were repeatedly exposed to the same strains. According to the model of strainspecific immunity to malaria [10,54], effective malaria immunity in low transmission areas may be achieved after a relatively small number of infective mosquito bites [41]. In addition, in areas with low levels of recombination, haplotypes may be maintained through multiple generations [41], continuously exposing their antigens to the human immune system [2]. In San Carlos, most participants were asymptomatic to the most frequent haplotype, H11, possibly due to the development of clinical immunity that was able to control the parasite density and prevent symptoms. Therefore, the limited genetic diversity and the low MOI in this low transmission area may still stimulate an efficient immune response [10] which could explain the common occurrence of asymptomatic and sub-patent infections.

In relatively isolated populations such as San Carlos, with few circulating clones, continued anti-malaria treatment/drug pressure may foster a genetic restructuring of the parasite population, favouring the spread of the drug resistance trait [9]. No indication of CQ resistance was found in the present study. However the frequent 
occurrence of recurrent infections with similar haplotypes after one month the radical treatment was given could be an early sign that the PQ is not working efficiently, probably due to resistant parasites or inefficient metabolic activation of the PQ by the participants. Further studies involving a larger number of endemic communities, with different rates of recurrences and parasite population structure, as well as the use of in vitro studies, resistance markers and whole parasite genome sequencing would give more insights on the rise and spread of resistant parasites and the parasitic genetic diversity.

\section{Conclusions}

Combining population genetic tools and space-time scan statistics allowed for an in-depth insight to the dynamics of $P$. vivax clones at individual and household level in this isolated community of the Peruvian Amazon. Space-time clustering of recurrent asymptomatic $P$. vivax infections with few specific clones reflects the highly focal transmission of the local parasite population favouring the development of clinical immunity by infected people. This type of setting could also be at high risk of selection and spread if drug resistant $P$. vivax clones are under high selective pressure. This type of information is highly valuable for further strengthening malaria elimination strategies in the Peruvian Amazon and should be extended to different endemic areas.

\section{Additional files}

Additional file 1: Population structure inferred by microsatellite genotyping of 136 Plasmodium vivax monoclonal infections using STRUCTURE. Panel $A$, using the method described elsewhere [32], the uppermost hierarchical level of structure was assumed, describing four populations (highest peak, $\mathrm{K}=4$ ). Two other peaks $(\mathrm{K}=6$ and $\mathrm{K}=9)$ are described with a lower likelihood. $K=6$ corresponded to the six

haplogroups defined by eBURST when the criteria of relatedness was increased to 14 loci instead of 11. Panel B illustrates the bar plot at $K=4$ with 136 samples being represented by a single vertical line divided into colours assigned to the population of origin. Each colour represents one population (A-green, B-blue, C-red, D-yellow), and the length of the coloured segment shows the estimated proportion of membership of that sample to each population.

Additional file 2: Characteristics of each malaria infection suffered by the $\mathbf{3 7}$ participants. $X=$ Initial infection in this study (D0). Green shading indicates a recurrent $P$. vivax infection. $P=$ patent infection. On the upper left corner of the cells is defined the haplotypes $\left(\mathrm{H}_{1} \ldots \mathrm{H} 2 \mathrm{O}\right)$ or one of the symbols explained below. $i=$ incomplete allele data, $m=$ mixed infections (not possible to differentiate the haplotypes). ind = haplotype within a mixed infection that cannot be determined. $n=P$. vivax sample that did not amplified by PCR with any of the microsatellites. ${ }^{\dagger}$ Patent infection detected by microscopy but no by ssPCR (MS genotyping was not done). ${ }^{\ddagger} \mathrm{Pv}$ positive by microscopy on D0 but filter paper D0 was not available. On day 1 was negative by SSPCR (MS genotyping was not performed).

\section{Competing interests}

The authors declare that they have no competing interests.

\section{Authors' contributions}

Conceived and designed the experiments: CD, VESC, PVdE, DG, HRF, ALC, AE, UDA. Performed the experiments: CD. Analysed the data: $C D, P V d E, A R, A E$, UDA. Contributed reagents/materials/ analysis tools: VESC, AR, ENA, DG, AR, AE. Wrote the paper: CD, VESC, DG, JPVG, AE, UDA. All authors read and approved the final manuscript.

\section{Acknowledgements}

We thank the San Carlos community and the field workers who participated in this study. The work was supported by the Belgian Agency Cooperation for Development, within the third framework agreement of the Institutional collaboration between the Institute of Tropical Medicine "Alexander von Humboldt" - Universidad Peruana Cayetano Heredia, Lima and the Institute of Tropical Medicine in Antwerp, Belgium (Project 95503). CD was supported by a VLIR-UOS (Vlaamse Interuniversitaire Road - University Development Cooperation, Belgium) for the data analysis and paper writing. The funders had no role in study design, data collection and analysis, decision to publish, or preparation of the manuscript.

\section{Author details}

${ }^{1}$ Unit of International Health, ESOC Department, Faculty of Medicine, University of Antwerp, Universiteitsplein 1, B-2610 Antwerp, Belgium. ${ }^{2}$ Laboratory of Malaria, Unit of Molecular Epidemiology, Institute of Tropical Medicine Alexander von Humboldt (IMTAvH)-Universidad Peruana Cayetano Heredia (UPCH), Av Honorio Delgado 430, Lima, Peru. ${ }^{3}$ Grupo de Estudio de Leishmaniasis y Malaria (GELM), ITMAvH-Universidad Peruana Cayetano Heredia (UPCH), Av Honorio Delgado 430, Lima 31, Peru. ${ }^{4}$ Malariology Unit, Department of Biomedical Sciences, Institute of Tropical Medicine, Antwerp, Nationalestraat 155, B-2000 Antwerp, Belgium. ${ }^{5}$ Departamento de Ciencias Celulares y Moleculares, Facultad de Ciencias y Filosofía- Universidad Peruana Cayetano Heredia, Av Honorio Delgado 430, Lima, Peru. 'Unit of Veterinary Biostatistics and Epidemiology, Institute of Tropical Medicine, Nationalestraat 155, B-2000 Antwerp, Belgium. ${ }^{7}$ Ministry of Health, Av 28 de Julio s/n, Punchana, Loreto, Peru. ${ }^{8}$ Department of Public Health, Institute of Tropical Medicine, Nationalestraat 155, B-2000 Antwerp, Belgium. ${ }^{9}$ Medical Research Council Unit, Fajara PO Box 273, Banjul, The Gambia.

Received: 4 September 2013 Accepted: 28 December 2013 Published: 6 January 2014

\section{References}

1. Price RN, Tjitra E, Guerra CA, Yeung S, White NJ, Anstey NM: Vivax malaria: neglected and not benign. Am J Trop Med Hyg 2007, 77:79-87.

2. Arnott A, Barry AE, Reeder JC: Understanding the population genetics of Plasmodium vivax is essential for malaria control and elimination. Malar J 2012, 11:14.

3. ORAS-CONHU: Situación de la Malaria en el Perú 2009. [http://www. orasconhu.org/documentos/2\%20lquitos\%20junio\%20-\%20Situacion\%20de \%20la\%20malaria\%202009.pdf]

4. Ruebush TK 2nd, Zegarra J, Cairo J, Andersen EM, Green M, Pillai DR, Marquino W, Huilca M, Arevalo E, Garcia C, Solary L, Kain KC: Chloroquineresistant Plasmodium vivax malaria in Peru. Am J Trop Med Hyg 2003, 69:548-552.

5. Van den Eede P, Soto-Calle VE, Delgado C, Gamboa D, Grande T, Rodriguez H, Llanos-Cuentas A, Anne J, D'Alessandro U, Erhart A: Plasmodium vivax sub-patent infections after radical treatment are common in Peruvian patients: results of a 1-year prospective cohort study. PLoS One 2011, 6:e16257.

6. Branch O, Casapia WM, Gamboa DV, Hernandez JN, Alava FF, Roncal N, Alvarez E, Perez EJ, Gotuzzo E: Clustered local transmission and asymptomatic Plasmodium falciparum and Plasmodium vivax malaria infections in a recently emerged, hypoendemic Peruvian Amazon community. Malar J 2005, 4:27.

7. Roshanravan B, Kari E, Gilman RH, Cabrera L, Lee E, Metcalfe J, Calderon M, Lescano AG, Montenegro SH, Calampa C, Vinetz JM: Endemic malaria in the Peruvian Amazon region of Iquitos. Am J Trop Med Hyg 2003, 69:45-52.

8. da Silva-Nunes M, Moreno M, Conn JE, Gamboa D, Abeles S, Vinetz JM, Ferreira MU: Amazonian malaria: asymptomatic human reservoirs, diagnostic challenges, environmentally driven changes in mosquito 
vector populations, and the mandate for sustainable control strategies. Acta Trop 2011, 121:281-291.

9. Hastings IM, D'Alessandro U: Modelling a predictable disaster: the rise and spread of drug-resistantmalaria. Parasitol Today 2000, 16:340-347.

10. Branch OH, Takala S, Kariuki S, Nahlen BL, Kolczak M, Hawley W, Lal AA: Plasmodium falciparum genotypes, low complexity of infection, and resistance to subsequent malaria in participants in the Asembo Bay Cohort Project. Infect Immun 2001, 69:7783-7792.

11. Ferreira MU, Karunaweera ND, da Silva-Nunes M, da Silva NS, Wirth DF, Hartl DL: Population structure and transmission dynamics of Plasmodium vivax in rural Amazonia. J Infect Dis 2007, 195:1218-1226.

12. Restrepo E, Imwong M, Rojas W, Carmona-Fonseca J, Maestre A: High genetic polymorphism of relapsing $P$. vivax isolates in northwest Colombia. Acta Trop 2011, 119:23-29.

13. Orjuela-Sanchez P, da Silva NS, da Silva-Nunes M, Ferreira MU: Recurrent parasitemias and population dynamics of Plasmodium vivax polymorphisms in rural Amazonia. Am J Trop Med Hyg 2009, 81:961-968.

14. Van den Eede P, Van der Auwera G, Delgado C, Huyse T, Soto-Calle VE, Gamboa D, Grande T, Rodriguez H, Llanos A, Anne J, Erhart A, D'Alessandro U: Multilocus genotyping reveals high heterogeneity and strong local population structure of the Plasmodium vivax population in the Peruvian Amazon. Malar J 2010, 9:151

15. Kosek M, Yori PP, Gilman RH, Calderon M, Zimic M, Chuquiyauri R, Jeri C, Pinedo-Cancino V, Matthias MA, Llanos-Cuentas A, Vinetz JM: High degree of Plasmodium vivax diversity in the Peruvian Amazon demonstrated by tandem repeat polymorphism analysis. Am J Trop Med Hyg 2012, 86:580-586.

16. Roper MH, Torres RS, Goicochea CG, Andersen EM, Guarda JS, Calampa C, Hightower AW, Magill AJ: The epidemiology of malaria in an epidemic area of the Peruvian Amazon. Am J Trop Med Hyg 2000, 62:247-256.

17. Grietens KP, Muela Ribera J, Soto V, Tenorio A, Hoibak S, Aguirre AR, Toomer E, Rodriguez H, Llanos Cuentas A, D'Alessandro U, Gamboa D, Erhart A: Traditional nets interfere with the uptake of long-lasting insecticidal nets in the peruvian Amazon: the relevance of net preference for achieving high coverage and use. PLoS One 2013, 8:e50294.

18. Grietens KP, Soto V, Erhart A, Ribera JM, Toomer E, Tenorio A, Montalvo TG, Rodriguez H, Cuentas AL, D'Alessandro U, Gamboa D: Adherence to 7-day primaquine treatment for the radical cure of $P$. vivax in the Peruvian Amazon. Am J Trop Med Hyg 2010, 82:1017-1023.

19. Gutierrez S, Arróspide N: Manual de Procedimientos de Laboratorio para el diagnóstico de Malaria. 2003 [http://www.ins.gob.pe/repositorioaps/0/4/jer/1/Antimalaricos/manualMALARIA.pdf]

20. Plowe CV, Djimde A, Bouare M, Doumbo O, Wellems TE: Pyrimethamine and proguanil resistance-conferring mutations in Plasmodium falciparum dihydrofolate reductase: polymerase chain reaction methods for surveillance in Africa. Am J Trop Med Hyg 1995, 52:565-568.

21. Rubio JM, Post RJ, van Leeuwen WM, Henry MC, Lindergard G, Hommel M Alternative polymerase chain reaction method to identify Plasmodium species in human blood samples: the semi-nested multiplex malaria PCR (SnM-PCR). Trans R Soc Trop Med Hyg 2002, 96(Suppl 1):S199-S204.

22. Karunaweera ND, Ferreira MU, Hartl DL, Wirth DF: Fourteen polymorphic microsatellite DNA markers for the human malaria parasite Plasmodium vivax. Mol Ecol Notes 2007, 7:172-175.

23. Van den Eede P, Erhart A, Van der Auwera G, Van Overmeir C, Thang ND, Hung le X, Anne J, D'Alessandro U: High complexity of Plasmodium vivax infections in symptomatic patients from a rural community in central Vietnam detected by microsatellite genotyping. Am J Trop Med Hyg 2010, 82:223-227.

24. Bruce MC, Macheso A, McConnachie A, Molyneux ME: Comparative population structure of Plasmodium malariae and Plasmodium falciparum under different transmission settings in Malawi. Malar J 2011, 10:38.

25. Anderson TJ, Su XZ, Bockarie M, Lagog M, Day KP: Twelve microsatellite markers for characterization of Plasmodium falciparum from finger-prick blood samples. Parasitology 1999, 119(Pt 2):113-125.

26. Arnaud-Haond S, Belhir K: Genclone: a computer program to analyse genotypic data, test for clonality and describe spatial clonal organization. Mol Ecol Notes 2007, 7:3.

27. Goudet J: FSTAT (Version 1.2): a computer program to calculate F-statistics. J Hered 1995, 86:485-486.

28. Rousset F: genepop'007: a complete re-implementation of the genepop software for Windows and Linux. Mol Ecol Resour 2008, 8:103-106.
29. Raymond M, Rousset F: An exact test for population differentiation. Evolution 1995, 49:4.

30. Haubold B, Hudson RR: LIAN 3.0: detecting linkage disequilibrium in multilocus data. Linkage Analysis. Bioinformatics 2000, 16:847-848.

31. Feil EJ, Li BC, Aanensen DM, Hanage WP, Spratt BG: eBURST: inferring patterns of evolutionary descent among clusters of related bacterial genotypes from multilocus sequence typing data. J Bacteriol 2004, 186:1518-1530.

32. Evanno G, Regnaut S, Goudet J: Detecting the number of clusters of individuals using the software STRUCTURE: a simulation study. Mol ECOl 2005, 14:2611-2620.

33. Bandelt $H J$, Forster $P$, Rohl A: Median-joining networks for inferring intraspecific phylogenies. Mol Biol Evol 1999, 16:37-48.

34. Kulldorff M: SaTScanTM: Software for the spatial, temporal, and space-time scan statistics. 2011 [http://www.satscan.org/]

35. Kulldorff M: A spatial scan statistics. Commun Stat: Theory Methods 1997, 26:16

36. Lee G, Yori P, Olortegui MP, Pan W, Caulfield L, Gilman RH, Sanders JW Delgado HS, Kosek M: Comparative effects of vivax malaria, fever and diarrhoea on child growth. Int J Epidemiol 2012, 41:531-539.

37. Chuquiyauri R, Paredes M, Penataro P, Torres S, Marin S, Tenorio A, Brouwer KC, Abeles S, Llanos-Cuentas A, Gilman RH, Kosek M, Vinetz JM: Socio-demographics and the development of malaria elimination strategies in the low transmission setting. Acta Trop 2012, 121:292-302.

38. Baird JK: Resistance to therapies for infection by Plasmodium vivax. Clin Microbiol Rev 2009, 22:508-534.

39. Villalobos-Salcedo JM, Tada MS, Kimura E, Menezes MJ, Pereira da Silva LH: In-vivo sensitivity of Plasmodium vivax isolates from Rond nia (western Amazon region, Brazil) to regimens including chloroquine and primaquine. Ann Trop Med Parasitol 2000, 94:749-758.

40. Chenet SM, Schneider KA, Villegas L, Escalante AA: Local population structure of Plasmodium: impact on malaria control and elimination. Malar J 2012, 11:412

41. Anderson TJ, Haubold B, Williams JT, Estrada-Franco JG, Richardson L, Mollinedo R, Bockarie M, Mokili J, Mharakurwa S, French N, Whitworth J, Velez ID, Brockman AH, Nosten F, Ferreira MU, Day KP: Microsatellite markers reveal a spectrum of population structures in the malaria parasite Plasmodium falciparum. Mol Biol Evol 2000, 17:1467-1482.

42. Imwong M, Nair S, Pukrittayakamee S, Sudimack D, Williams JT, Mayxay M, Newton PN, Kim JR, Nandy A, Osorio L, Carlton JM, White NJ, Day NP, Anderson TJ: Contrasting genetic structure in Plasmodium vivax populations from Asia and South America. Int J Parasitol 2007, 37:1013-1022.

43. Rezende AM, Tarazona-Santos E, Couto AD, Fontes CJ, De Souza JM, Carvalho LH, Brito CF: Analysis of genetic variability of Plasmodium vivax isolates from different Brazilian Amazon areas using tandem repeats. Am J Trop Med Hyg 2009, 80:729-733.

44. Chuquiyauri R, Penataro P, Brouwer KC, Fasabi M, Calderon M, Torres S, Gilman RH, Kosek M, Vinetz JM: Microgeographical differences of Plasmodium vivax relapse and re-infection in the Peruvian Amazon. Am J Trop Med Hyg 2013, 89:326-338.

45. Abeles SR, Chuquiyauri R, Tong C, Vinetz JM: Human host-derived cytokines associated with Plasmodium vivax transmission from acute malaria patients to Anopheles darlingi mosquitoes in the Peruvian Amazon. Am J Trop Med Hyg 2013, 88:1130-1137.

46. Clark EH, Silva CJ, Weiss GH, Li S, Padilla C, Crompton PD, Hernandez JN, Branch $\mathrm{OH}$ : Plasmodium falciparum malaria in the low transmission Peruvian Amazon is associated with immunologic memory. Infect Immun 2012, 80:1583-1592.

47. Sutton PL, Neyra V, Hernandez JN, Branch OH: Plasmodium falciparum and Plasmodium vivax infections in the Peruvian Amazon: propagation of complex, multiple allele-type infections without super-infection. Am $J$ Trop Med Hyg 2009, 81:950-960.

48. Gunawardena S, Karunaweera ND, Ferreira MU, Phone-Kyaw M, Pollack RJ, Alifrangis M, Rajakaruna RS, Konradsen F, Amerasinghe PH, Schousboe ML, Galappaththy GN, Abeyasinghe RR, Hartl DL, Wirth DF: Geographic structure of Plasmodium vivax: microsatellite analysis of parasite populations from Sri Lanka, Myanmar, and Ethiopia. Am J Trop Med Hyg 2010, 82:235-242.

49. Karunaweera ND, Ferreira MU, Munasinghe A, Barnwell JW, Collins WE, King CL, Kawamoto F, Hartl DL, Wirth DF: Extensive microsatellite diversity in the human malaria parasite Plasmodium vivax. Gene 2008, 410:105-112.

50. Levinson G, Gutman GA: Slipped-strand mispairing: a major mechanism for DNA sequence evolution. Mol Biol Evol 1987, 4:203-221. 
51. Imwong M, Snounou G, Pukrittayakamee S, Tanomsing N, Kim JR, Nandy A, Guthmann JP, Nosten F, Carlton J, Looareesuwan S, Nair S, Sudimack D, Day NP, Anderson TJ, White NJ: Relapses of Plasmodium vivax infection usually result from activation of heterologous hypnozoites. I Infect Dis 2007, 195:927-933.

52. Markus MB: Dormancy in mammalian malaria. Trends Parasitol 2012, 28:39-45.

53. Wykes MN, Kay JG, Manderson A, Liu XQ, Brown DL, Richard DJ, Wipasa J, Jiang SH, Jones MK, Janse CJ, Waters AP, Pierce SK, Miller LH, Stow JL, Good MF: Rodent blood-stage Plasmodium survive in dendritic cells that infect naive mice. Proc Natl Acad Sci U S A 2011, 108:11205-11210.

54. Gupta S, Maiden MC, Feavers IM, Nee S, May RM, Anderson RM: The maintenance of strain structure in populations of recombining infectious agents. Nat Med 1996, 2:437-442.

doi:10.1186/1475-2875-13-8

Cite this article as: Delgado-Ratto et al:: Population structure and spatio-temporal transmission dynamics of Plasmodium vivax after radical cure treatment in a rural village of the Peruvian Amazon. Malaria Journal 2014 13:8

\section{Submit your next manuscript to BioMed Central and take full advantage of:}

- Convenient online submission

- Thorough peer review

- No space constraints or color figure charges

- Immediate publication on acceptance

- Inclusion in PubMed, CAS, Scopus and Google Scholar

- Research which is freely available for redistribution 